\section{¿Positiva o negativa para la salud poblacional?: Respuestas al debate sobre las dos caras de cómo una crisis económica afecta la salud a partir del caso español}

\author{
Positive or negative for population \\ health? Responses to the debate \\ on the two sides of how an \\ economic crisis affects health \\ based on the Spanish case
}

Jeroen Spijker ${ }^{1}$, Jordi Gumà

'Doctor en Ciencias Espaciales (especialización Demografía). Investigador "Ramón y Cajal", Centro de Estudios Demográficos, Bellaterra, España. $\square$ (ID)

${ }^{2}$ Doctor en Demografía. Investigador "Juan de la Cierva", Research and Expertise Centre for Survey Methodology, Universitat Pompeu Fabra, Barcelona, España. $\bowtie$ (iD)

doi: 10.18294/sc.2018.2103

\section{Respuesta a:}

Rita Barradas Barata do

James Macinko doi

Arthur L. Frank do

Maurício L. Barreto do:

José A. Tapia Granados
Los resultados de nuestro estudio ${ }^{(1)}$ han demostrado que la evolución de los indicadores de salud durante los años de crisis no modificaron las grandes tendencias de mejoras en la salud global de la población, lo cual a priori podría resultar contraintuitivo. De hecho, la hipótesis de partida en nuestra investigación era que las diferencias en el nivel de salud entre personas de mayor o menor nivel educativo o entre empleados y desempleados aumentarían como consecuencia de la crisis. Sin embargo, los resultados no corroboran esta hipótesis ya que la proporción de personas que perciben su propia salud como mala disminuyó en todos los grupos estudiados de hombres y mujeres de 30 a 59 años en la primera fase de la crisis analizada (entre 2006 y 2010) y en la mayoría de los grupos -excepto unos pocos en los que aumenta muy débilmente- en la segunda fase de la crisis (entre 2010 y 2014).

A la luz de estos resultados, tal y como apunta Tapia Granados (2), se podría concluir que dicho patrón no es compatible con un efecto nocivo de la crisis sobre la salud y que, por el contrario, se debería apuntar a un efecto beneficioso. Sin embargo, a pesar de lo apuntado anteriormente hemos sido muy cautos en realizar tal afirmación, y esto se debe a diversas razones. En primer lugar, aunque podríamos haber destacado más el hecho de que la salud autopercibida mejoró bastante durante el primer periodo de la crisis, éramos y seguimos siendo reacios a constatar firmemente que una crisis beneficia la salud debido a los efectos adversos que se han observado en trabajos anteriores, como el aumento del consumo de medicamentos o el incremento de los problemas de salud mental ${ }^{(3,4,5)}$. Segundo, para poder asegurar que una crisis es realmente beneficiosa para la salud, sus efectos positivos deberían perdurar, algo que no observamos en nuestros resultados ya que en el segundo periodo de crisis se ve un claro estancamiento en la mejora de la salud, sobre todo entre las mujeres y los grupos socioeconómicos más altos, sin olvidar los hombres monoparentales. Sería interesante, para futuras investigaciones, averiguar por qué justamente personas con un nivel educativo más alto o con una mayor capacidad económica para llegar a fin de mes se vieron más afectadas durante el segundo periodo de la crisis a pesar de que, como menciona Barradas Barata ${ }^{(6)}$, la segunda oleada de la crisis estaba marcada por un alto impacto social que, entre otras consecuencias, mostró un incremento de la tasa de desempleo, una creciente dificultad de los jóvenes para introducirse en el mercado laboral, y políticas de austeridad que reducen los beneficios sociales. La clave 
podría encontrarse en el endurecimiento de las condiciones de trabajo y el consiguiente aumento en el nivel de presión sobre aquellas personas con un cierto nivel de responsabilidad laboral, algo que no analizamos y que también podría estar detrás de la falta de mejora entre las mujeres durante el segundo periodo. De hecho, Tapia Granados ${ }^{(2)}$ también apunta a las condiciones laborales como una posible razón para explicar el posible beneficio sobre la salud poblacional durante períodos de inestabilidad económica, pero señalando, en este caso, a aquellos grupos con un estatus socioeconómico más bajo, para los cuales dejar de trabajar implicaría no estar expuestos en su día a día a un contexto potencialmente pernicioso para su estado de salud. Esto último iría en el mismo sentido que algunas investigaciones que apuntan que los periodos de crisis económica tienen un efecto de mejora sobre ciertos comportamientos relacionados con la salud, como el aumento de las horas de sueño o de tiempo libre, que pueden destinarse a la realización de actividades saludables (como el ejercicio físico), un consumo menor de alimentos poco saludables y de alcohol y tabaco por la contracción de los presupuestos familiares, o la reducción del tiempo de conducción con un descenso de la accidentalidad por tráfico.

De todos modos, estos posibles efectos beneficiosos se localizarían sobre todo en el corto plazo, aunque los beneficios de los cambios de hábitos se podrían también alargar en el tiempo si estos se mantienen. Sin embargo, no se pueden obviar las evidencias encontradas recientemente, gracias al acceso a datos con un mayor recorrido temporal, sobre los efectos negativos que las trayectorias laborales discontinuas han mostrado tener sobre la salud en el medio y largo plazo ${ }^{(7,8)}$, motivo que refuerza nuestra cautela en la interpretación de los "beneficios" sobre la salud de la crisis económica. Actualmente, se está produciendo un interesante debate sobre si una situación socioeconómica adversa tiene o no efectos acumulativos a lo largo de la trayectoria vital de las personas ${ }^{(8)}$, lo cual en el caso de ser cierto apuntaría a que los verdaderos efectos sobre la salud de las fluctuaciones macroeconómicas solamente serían observables en el largo plazo. En este sentido, debemos seguir monitoreando la salud de la población y resulta necesario que las instituciones refuercen las políticas de protección y cohesión social, especialmente, entre las personas en situaciones socioeconómicas desfavorecidas, y mejoren la cobertura, el acceso y la calidad del sistema de salud, al tiempo que se diseñen políticas para promover la equidad en la salud. Por ejemplo, ahora que estamos en una fase de recuperación económica, sería importante apoyar programas y actividades orientados a promover la actividad física en personas mayores y monitorear bien su evolución (es decir, por edad, sexo y categorías socioeconómicas).

Además, tal y como señalamos en el artículo, una parte de las mejoras en la salud autopercibida de las personas desocupadas, sobre todo entre los hombres, se explicaría por una mayor diversidad en el perfil sociodemográfico, como resultado de su incremento en términos absolutos. De este modo, mientras que antes las personas desocupadas mostraban un perfil más concreto y caracterizado por una situación general de mayor vulnerabilidad (niveles educativos bajos, situaciones de mala salud que dificultaban el acceso al empleo, etc.), en este periodo de crisis, el perfil de las personas desempleadas se diversificó, incluyendo grupos que anteriormente no habían sido considerados como potencialmente vulnerables. Por lo tanto, nos aventuramos a predecir que una vez que la población desocupada se reduzca a niveles precrisis, habrá otra vez una relación más clara con la mala salud.

Quisiéramos también destacar el peso que se le ha dado en el debate sobre nuestro trabajo a dos variables eminentemente demográficas como son la cohorte de nacimiento y el género. Barradas Barata ${ }^{(6)}$ señala la importancia del efecto educativo en las mejoras de la salud autopercibida de la población a lo largo del tiempo, ya que las generaciones más antiguas tienen proporciones muy bajas de población con estudios superiores, que se concentran en la población masculina, mientras que, a medida que 
observamos generaciones más jóvenes, esta proporción aumenta por la expansión educativa, en gran parte protagonizada por la población femenina. De este modo, dos poblaciones dentro del mismo grupo de edad 30-59, separadas por una distancia temporal de ocho años, muestran perfiles educativos diferentes como, por ejemplo, un aumento de 11 puntos porcentuales de mujeres con estudios universitarios, como muestra la Tabla 1 en nuestro estudio(1). Por lo tanto, dado que quienes tienen un nivel educativo alto declaran tener, como promedio, mejor salud, una parte de la mejora de la salud autopercibida en el conjunto de la población en los diferentes años -con el periodo de crisis incluido- se explicaría por la cambiante estructura de la población a nivel educativo.

En cuanto a las diferencias de género, Frank señala en el debate ${ }^{(9)}$ que, en general, a las mujeres se les paga menos que a los hombres. Como resultado, tenemos el problema continuo y creciente del estancamiento o incluso disminución del ingreso familiar, debido a las presiones inflacionarias y los cambios en el mercado laboral, con empleos de menor remuneración que reemplazan a los empleos mejor pagados, múltiples empleos a tiempo parcial que reemplazan empleos a tiempo completo y empleos con malas condiciones laborables. A medida que el deterioro de las condiciones de empleo se generaliza, especialmente entre las cohortes más jóvenes, los esfuerzos en salud pública deberían tener entre sus prioridades la mejor prevención de riesgos y promoción de la salud en las etapas más tempranas del ciclo de vida para minimizar los efectos adversos para la salud en las edades más avanzadas.

Respecto a otros posibles mecanismos detrás de nuestros resultados, existen estudios previos que sugieren que el efecto amortiguador del apoyo social y la solidaridad familiar reduce el impacto de la recesión económica sobre la salud, lo que podría explicar la falta de empeoramiento de la salud autopercibida en nuestra población de estudio, sobre todo, para los hombres. Otros estudios destacan la influencia positiva del mantenimiento de las prestaciones sociales y que, a pesar de los recortes y copagos introducidos, los dispositivos sanitarios asistenciales públicos siguen siendo de acceso universal y gratuito.

Otro motivo para mantener la cautela en nuestras interpretaciones se basa en que se debe tener en cuenta que los resultados de toda investigación cuantitativa, como es nuestro caso, se muestran dependientes de las metodologías y los indicadores empleados para medir los niveles de salud de la población y los cambios socioeconómicos. En nuestro trabajo tuvimos que tomar una serie de decisiones que tal vez requieren de una explicación más detallada. Por ejemplo, la exclusión de los nacidos fuera de España, señalada por Tapia Granados ${ }^{(2)}$, se debe al hecho de tratarse de un grupo muy heterogéneo en cuanto a su procedencia y duración en España. De hecho, el perfil sociodemográfico del contingente de inmigrantes establecidos en España ha cambiado en forma drástica, como consecuencia precisamente del reciente periodo de recesión económica. Aquellos con una situación más vulnerable decidieron probar suerte en otros países del continente europeo, al ser las primeras víctimas de la desaparición masiva de puestos de trabajo ${ }^{(10)}$. Por tanto, se trataría de un tema que debería ser investigado de manera específica y que, en el caso de haberse incluido en nuestro análisis, podría haber llevado a resultados difíciles de explicar si no considerasen de manera detallada las características demográficas y socioeconómicas de los inmigrantes en los diferentes momentos en el tiempo.

Del mismo modo, aunque la encuesta distingue entre cinco posibles escalas de salud, nosotros decidimos dicotomizar las categorías como muy buena/buena y regular/mala/muy mala. No obstante, tal como señala Macinko(11), al agrupar las posibles respuestas, perdemos el detalle sobre la verdadera magnitud del cambio en el resultado. Quizá los cambios en las subpoblaciones que informaron salud autopercibida mala o muy mala implicaron un desplazamiento de 3 o 4 puntos, pasando de salud muy buena a salud muy mala, o un cambio más pequeño, pasando de buena a regular. En ambos casos, 
para nosotros tenía el mismo significado (de tener buena a tener no buena salud). Aunque la ventaja de dicotomizar variables es simplificar el análisis estadístico y facilitar tanto la interpretación como la presentación de los resultados, somos conscientes de la pérdida de detalle en la información. Debido a ello, futuras investigaciones deberían analizar con más detalle los cambios de salud durante periodos de crisis más allá del mero hecho de tener buena salud o no.

Además, se debe tener en cuenta que trabajar con muestras de la EU-SILC separadas por un periodo de cuatro años es equivalente a utilizar una encuesta transversal con muestras independientes, mientras que, por ejemplo, el caso de las enfermedades crónicas suele ser el resultado de una acumulación de exposiciones en el transcurso de la vida, exposiciones que además tienden a ser mayores entre las clases socioeconómicas más bajas ${ }^{(12)}$. Por tanto, aunque a corto plazo la crisis parezca haber beneficiado más la salud de las personas de 30-59 años en España que pertenecen a clases menos favorecidas, ello no garantiza que la brecha siga reduciéndose en el futuro. Por este motivo, sería conveniente que futuras investigaciones sobre este interesante tema se basen en información longitudinal que haya observado a las mismas personas en periodos anteriores, durante y después de la crisis económica. Para finalizar, señalar que nuestro trabajo es otro pasito más en el sentido de entender mejor la complejidad de la relación entre los factores contextuales y la salud de los individuos. Queda claro, gracias al rico debate que ha generado nuestro trabajo, que aún queda por recorrer un largo camino hasta la completa comprensión de cómo se articula esta relación en el corto, medio y largo plazo. En este sentido, estamos totalmente de acuerdo con Barreto ${ }^{(13)}$ que el relato no será completo hasta que se pueda contar con la experiencia al respecto en América Latina, un contexto tan o más rico que el observado en el continente europeo debido a la diversidad social y económica.

\section{AGRADECIMIENTOS}

Esta investigación pudo ser realizada gracias al Programa CERCA de la Generalitat de Catalunya y los programas $\mathrm{I}+\mathrm{D}+\mathrm{i}$ del Ministerio de Economía y Competitividad de España Ramón y Cajal (RYC-201314851) para Jeroen Spijker y Juan de la Cierva (FJCl2015-25066) y para Jordi Gumà. Además, este trabajo se ha realizado en el marco del proyecto: "Anàlisi demogràfica de les desigualtats socioeconòmiques de la salut a Catalunya en el context de la darrera crisi econòmica: gènere, generació i territori", financiado por RecerCaixa (2015 ACUP 00108) y dirigido por el doctor Jeroen Spijker.

\section{REFERENCIAS BIBLIOGRÁFICAS}

1. Spijker J, Gumà J. El efecto de la crisis económica sobre la salud en España según el nivel educativo y la relación con la actividad: iimporta también la duración de la crisis? Salud Colectiva. 2018;14(4):655-670. doi: 10.18294/sc.2018.1297.

2. Tapia Granados JA. Spijker y Gumà confirman que durante la crisis económica en España hubo una mejora de indicadores de salud. Salud Colectiva. 2018;14(4):685687. doi: 10.18294/sc.2018.1987.

3. Barr B, Taylor-Robinson D, Scott-Samuel A, McKee M, Stuckler D. Suicides associated with the 2008-10 economic recession in England: time trend analysis. BMJ. 2012;345:e5142.

4. Virtanen $M$, Kivimäki $M$, Elovainio $M$, Vahtera J, Kokko K, Pulkkinen L. Mental health and hostility as predictors of temporary employment: evidence from two prospective studies. Social Science \& Medicine. 2005;61(10):2084-2095.

5. Spijker J, Bayona J, Medina A, Menacho T. Atles de salut de la població de 50 i més anys a Catalunya. Bellaterra: Centre d'Estudis Demogràfics; 2018.

6. Barradas Barata R. Autopercepción del estado de salud, las desigualdades en salud y la crisis económica: el caso de España. Salud Colectiva. 2018;14(4):671-674. doi: 10.18294/sc.2018.1984.

7. Arpino B, Gumà J, Julià A. Early-life conditions and health at older ages: The mediating role of educational attainment, family and employment trajectories. PloS One. 2018;13(4):e0195320.

8. Singh-Manoux A, Ferrie JE, Chandola T, Marmot M. Socioeconomic trajectories across the life course and health outcomes in midlife: evidence for the accumulation hypothesis? International Journal of Epidemiology. 2004;33(5):1072-1079.

9. Frank AL. Salud, seguridad y empleo. Salud Colectiva. 2018;14(4):679-680. doi: 10.18294/sc.2018.1989.

10. Medina Moral E, Herrarte Sánchez A, Vicéns Otero J. Inmigración y desempleo en España: impacto de la 
crisis económica. Información Comercial Española, ICE: Revista de Economía. 2010;854:37-48.

11. Macinko J. Austeridad, inequidad y resiliencia en España. Salud Colectiva. 2018;14(4):675-678. doi: 10.18294/ sc.2018.1988.

12. Blane D. The life course, the social gradient and health. En: Marmot MG, Wilkinson RG, (eds.). Social determinants of health. Oxford: Oxford University Press; 1999.

13. Barreto ML. La austeridad visita a América Latina: Aprendizajes de la reciente experiencia europea sobre el estudio de sus efectos en la salud. Salud Colectiva. 2018;14(4):681-684. doi: 10.18294/sc.2018.1990.

\section{FORMA DE CITAR}

Spijker J, Gumà J. ¿Positiva o negativa para la salud poblacional?: Respuestas al debate sobre las dos caras de cómo una crisis económica afecta la salud a partir del caso español. Salud Colectiva. 2018;14(4):689-693. doi: $10.18294 /$ sc. 2018.2103.

\section{(C) Esta obra está bajo una licencia Creative Commons Atribución-NoComercial 4.0 (c) $\mathrm{BC}$ Internacional.}

\section{COMISIÓN DE INVESTIGACIONES CIENTIFICAS Ministerio de Ciencia, Tecnologia e Innovación \\ Buenos Aires Provincia}

Este artículo fue traducido al inglés por Vanessa Di Cecco con fondos del Subsidio para Publicaciones de Divulgación Científica (Acta No 1459/17) de la Comisión de Investigaciones Científicas de la provincia de Buenos Aires.

http://dx.doi.org/10.18294/sc.2018.2103 